risk of developing BV $\quad(\mathrm{AHR}=0.99 \quad 95 \%$ CI $\quad 0.098-1.00$, $\mathrm{p}=0.038)$. The vaginal microbiome of women who developed BV was characterised by high microbial diversity and less stability compared to controls $(p=0.04)$.

Conclusion In a cohort that was designed carefully to study incident BV, lower abundance of L. crispatus, increased abundance of $G$. vaginalis, and detection of BVAB TM7 were significantly associated with development of BV, after adjusting for bacterial species and sexual behaviour. Increased vaginal microbial diversity, decreased stability and exposure to new sexual partners were also associated with the development of $\mathrm{BV}$ in WSW. Incident BV may result from sexual exchange of key BV-associated bacteria such as $G$. vaginalis which could destabilise the microbial ecology through displacement of beneficial bacteria such as $L$. crispatus.

\section{P2.09 KNOWLEDGE OF MICROBICIDE AMONG COMMERCIAL SEX WORKERS IN NAIROBI KENYA}

${ }^{1}$ Charles Salil, ${ }^{2}$ E Jeptanui. ${ }^{1}$ Information Centre for HIV Prevention, Kenya; ${ }^{2}$ Moi Teaching and Refferal Hospital, Kenya

\subsection{6/sextrans-2017-053264.186}

Introduction To determine the current knowledge of Microbicide and its future usage among Commercial Sex Workers who are exposed to unproductive sex.Also to assess how Microbicide could affect their sexual behaviours.

Methods Self administered Questionnaires among 55 respondents were used in a cross sectional way to investigate how they will take Microbicide as a preventive measure. This survey was conducted in the month of August-September 2015.

Results $58.5 \%$ of the respondents have never heard of Microbicide while $41.5 \%$ of the respondents have heard of Microbicide. The respondents in both cases were sexually active. The acceptance of Microbicide among the respondents was $80.2 \%$ if administered by a medical personnel advise while $19.8 \%$ of the respondents were ready to use immediately without an advice of a medical personnel advise.2.1\%of the respondents were not sure whether to use Microbicide or not since they didn't know the side effects of the Product and its reliability on prolonged usage and they preferred condom use ALWAYS. Conclusion To meet Commercial Sex Workers (CSW) needs, more information and knowledge should be made available to them through awareness creation campaigns, web pages of Commercial sex workers etc. Also easy to use methods of Microbicide should also be put in place which is to be conducted by a medical expert to avoid misuse of the Product. However, for most of the respondents, Microbicide is most acceptable method of Prevention and they could use more frequently than condom usage.

\section{P2.10 WHAT IS THE ROLE OF PAIRED RPR TESTING IN THE DIAGNOSIS OF SYPHILIS REINFECTION AND THE FOLLOW UP OF SYPHILIS?}

Chris Kenyon, Kara Osbak, Marjan Van Esbroeck. Institute of Tropical Medicine, Antwerp, Belgium

10.1136/sextrans-2017-053264.187

Introduction Syphilis reinfections are playing an increasing role in syphilis transmission in a number of populations. The assessment of reinfection and response to treatment depends on accurately measuring intraindividual changes in non-treponemal tests (delta-NTTs). In a 0 to 6 month delta-RPR determined by routine RPR testing (RT), samples would be tested 6 months apart with differences in reagent batches, environmental temperatures and observers all leading to measurement errors. We hypothesised that conducting paired RPR (PT) would enable a more accurate determination of delta-RPR than RT.

Methods 120 patients with a new diagnosis of syphilis were followed up at $0,3,6,9,12,18$ and 24 months with RPRs performed via RT at each study visit and at any suspected reinfection. RPR PT was performed at 0 and 6 months and at any suspected reinfection.

Results The quantitative agreement +/-1 dilution among PT and RT was $97.4 \%$. There was no difference in the proportion with an incomplete serological response at 6 months: 21 (19.4\%) and $19(17.6 \%)$ according to PT and RT, respectively $(p=0.726)$. There was no statistically significant difference between 0 to 6 month delta-RPR as determined by PT and $\mathrm{RT}$ in predicting seroresponse at 12 months $(86.1 \%$ and $91.6 \%$ agreement with 12 month classification, respectively, $\mathrm{p}=0.262$. PT did not reduce the numbers of those classified with asymptomatic reinfections.

Conclusion In our setting routine PT is unlikely to be worth the considerable effort and cost it entails. Further research is required to assess its utility in specific circumstances.

\section{P2.11 ASSOCIATION BETWEEN GENITAL HERPES SIMPEX VIRUS TYPE-2 SHEDDING AND PRESENCE OF BACTERIAL VAGINOSIS-ASSOCIATED BACTERIA}

${ }^{1}$ Christine Johnston, ${ }^{2}$ Amalia Magaret, ${ }^{1}$ Krista Yuhas, ${ }^{3}$ Sujatha Srinivasan, 'Sean Proll, ${ }^{1}$ James P Hughes, ${ }^{3}$ Christina M Kohler, ${ }^{1}$ Dana Varon, ${ }^{1}$ Thepthara N Pholsena, ${ }^{4}$ Jeanne M Marrazzo, ${ }^{2}$ David N Fredricks, ${ }^{2}$ Anna Wald. ${ }^{1}$ University of Washington, USA; ${ }^{2}$ University of Washington, Fred Hutchinson Cancer Research Centre, USA; ${ }^{3}$ Fred Hutchinson Cancer Research Centre, USA; ${ }^{4}$ University of Alabama, USA

\subsection{6/sextrans-2017-053264.188}

Introduction Herpes simplex virus type 2 (HSV-2) infection increases the risk of bacterial vaginosis (BV). We hypothesised that the biologic mechanism of this association is that genital HSV-2 shedding increases inflammation, resulting in increased presence and quantity of BV-associated bacteria (BVAB).

Methods HSV-2 seropositive women with a clinical history of $\mathrm{BV}$ in the past 12 months collected daily genital swabs for HSV detection and vaginal swabs for Nugent score and analysis of the microbiome for 28 days. BV was defined as Nugent score $>=7$. Quantitative PCR (qPCR) with species specific primers for Lactobacillus crispatus, L. iners, L. jensenii, Gardnerella vaginalis, Megasphaera and BVAB-2 were performed. HSV was detected using real-time qPCR. The presence of each bacterial species was compared on days with and without HSV shedding using Poisson regression.

Results Forty-eight women (median age 40; 48\% white) with a median of 2 genital HSV-2 recurrences in the prior year (range 0-12) were enrolled for a total of 1277 days of observation. Genital HSV shedding was detected on $134(10 \%)$ days. Of 960 days with Nugent score available, BV was present on $351(37 \%)$ days. The risk of BV was not significantly different in the presence of HSV shedding $(\mathrm{RR}=0.84$, $95 \% \mathrm{CI}=0.66-1.07)$. Several bacterial species appeared to be detected more frequently on days with HSV shedding as 